
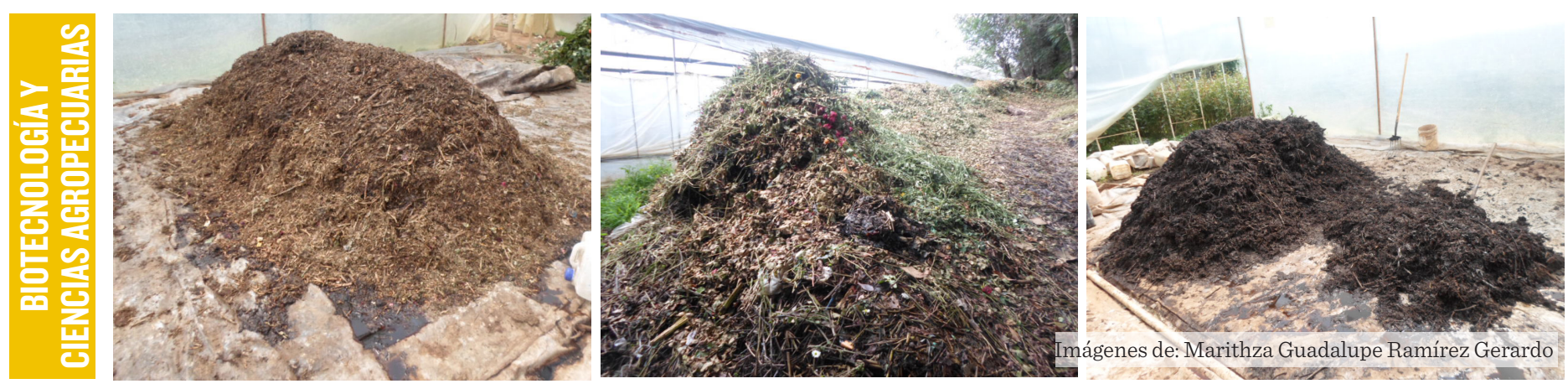

\title{
Caracterización de abonos orgánicos aplicados a cultivos florícolas en el sur del Estado de México
}

\section{Characterization of organic fertilizers applied to flower crops in the south of the State of Mexico}

\author{
Marithza Guadalupe Ramírez-Gerardo1*, Sergio Vázquez-Villegas', Gloria Isabel Méndez-Gómez', Jaime Mejía-Carranza²
}

\section{RESUMEN}

Las ventajas de incorporar abonos orgánicos (AO) disminuyen cuando sus propiedades físicas, químicas y microbiológicas no son las adecuadas. El objetivo de la presente investigación fue caracterizar abonos orgánicos destinados a suelos florícolas y analizar las repercusiones de su aplicación. Se determinaron las propiedades de 10 AO: potencial de hidrógeno $(\mathrm{pH})$, conductividad eléctrica $(\mathrm{CE})$, materia orgánica (MO), contenido de nitrógeno, fósforo, potasio y nitratos. Se utilizó un diseño completo al azar y análisis de varianza ( $\mathrm{P} \leq 0.05)$ para evaluar el índice germinativo (IG) en semillas de rábano y maíz, con lixiviados de $\mathrm{AO}$ al $5 \%$, así como la actividad microbiana de tres AO incorporados al suelo en incubaciones aerobias. El $\mathrm{pH}$ promedio fue de $8.2 \pm 0.81$, y el intervalo de la $\mathrm{CE}$ en $\mathrm{AO}$ fue de $0.15 \mathrm{dS} / \mathrm{m}$ a $6.7 \mathrm{dS} / \mathrm{m}$, mientras que el de la MO fue de $28.8 \%$ a $80 \%$. El IG fue diferente estadísticamente $(\mathrm{P}<0.05)$ en cada especie de semilla. Asimismo, la incorporación de AO incrementó significativamente $(\mathrm{P}<0.05)$ la actividad microbiana del suelo. Se concluye que la heterogeneidad de la materia prima genera una gran variabilidad en las características de los $\mathrm{AO}$, sin embargo, en todos los casos, su aplicación mejoró las propiedades de los suelos florícolas.

PALABRAS CLAVE: composta, lombricomposta, materia orgánica, relación carbono/nitrógeno $(\mathrm{C} / \mathrm{N})$.

\section{ABSTRACT}

The advantages of incorporating organic fertilizers (OF) decrease when their physical, chemical and microbiological properties are not adequate. The objective of this research was to characterize organic fertilizers intended for floricultural soils and analyze the effects of their application. The properties of 10 OF were determined: hydrogen potential $(\mathrm{pH})$, electrical conductivity (EC), organic matter (OM), content of nitrogen, phosphorus, potassium and nitrates. A completely randomized design and analysis of variance $(P \leq 0.05)$ were used to evaluate, the germination index (GI) in radish and corn seeds, with leachates of $\mathrm{OF}$ at $5 \%$, as well as the microbial activity of three $\mathrm{OF}$ incorporated into the soil in aerobic incubations. The average $\mathrm{pH}$ was $8.2 \pm 0.81$, and the interval of $\mathrm{EC}$ in $\mathrm{OF}$ ranged from $0.15 \mathrm{dS} / \mathrm{m}$ to $6.7 \mathrm{dS} / \mathrm{m}$, whereas that of the OM was from $28 \%$ to $80 \%$. The GI was statistically different $(\mathrm{P}<0.05)$ in each seed species. Likewise, the incorporation of $\mathrm{OF}$ significantly increased $(\mathrm{P}<0.05)$ the microbial activity of the soil. It is concluded that the heterogeneity of the raw material generates a great variability in the characteristics of the OF; however, in all cases, its application improved the properties of the floricultural soils.

KEYWORDS: compost, lombricompost, organic matter, carbon/nitrogen ratio $(\mathrm{C} / \mathrm{N})$.

*Correspondencia: maritthza@gmail.com/ Fecha de recepción: 4 de enero de 2021/Fecha de aceptación: 21 de abril de 2021/Fecha de publicación: 2 'r de julio de 2021.

${ }^{1}$ Tecnológico Nacional de México, Tecnológico de Estudios Superiores de Villa Guerrero, División de Ingeniería en Innovación Agrícola Sustentable, carretera federal Toluca-Ixtapan de la Sal km 64.5, La Finca, Villa Guerrero, Estado de México, México, C. P. 51767. ㄹniversidad Autónoma del Estado de México, Centro Universitario Tenancingo, Tenancingo, Estado de México, México. 


\section{INTRODUCCIÓN}

La agricultura convencional se caracteriza por el empleo de agroquímicos como fertilizantes, plaguicidas y herbicidas de origen sintético, que permiten alcanzar altos rendimientos, sin embargo, esta forma de producción está vinculada al deterioro físico, químico y biológico del suelo (Sakin y col., 2018). El municipio de Villa Guerrero, en el Estado de México, México, forma parte del corredor florícola más importante del país, con una producción intensiva de flores de corte, cuyos sistemas productivos se caracterizan por tener una alta demanda de nutrientes, como nitrógeno $(\mathrm{N})$, fósforo $(\mathrm{P})$ y potasio $(\mathrm{K})$ (Cabrera y col., 2017) que los floricultores incorporan como fertilizantes sintéticos, acción que impacta de manera negativa a los suelos, ya que con el tiempo genera problemas de salinidad, empobrecimiento de nutrientes y disminución de actividad microbiana (Bonanomi y col., 2020; Yáñez-Ocampo y col., 2020). Actualmente se han comenzado a utilizar diversas fuentes de materia orgánica (MO), como estiércoles de bovino, equino y gallinaza de animales, que tiene el propio productor, humus de lombriz, composta, pajas y desechos de cultivos, en cantidades variables, con el objetivo de disminuir principalmente costos en la compra de fertilizantes sintéticos, pero también para mantener la salud y calidad de los suelos y mejorar su productividad; sin embargo, se desconocen las propiedades físicas, químicas y microbiológicas de los abonos orgánicos $(\mathrm{AO})$ que incorporan.

La aplicación de los AO al suelo se considera una práctica agrícola sustentable, porque contribuye a mantenerlos sanos y fértiles (Scotti y col., 2015; Vilkiené y col., 2016; Bonanomi y col., 2020) debido a que son fuente de nutrimentos, principalmente $\mathrm{N}, \mathrm{P}, \mathrm{K}$, calcio (Ca) y magnesio $(\mathrm{Mg})$, y porque mejoran las características físicas del suelo, como la textura, densidad aparente, formación y estabilidad de agregados, y características químicas como potencial de hidrógeno $(\mathrm{pH})$, conductividad eléctrica $(\mathrm{CE})$, saturación de bases intercambiables, aporte de MO y nutrimentos del suelo y, en consecuencia, la productividad de los cultivos (Dikr y Belete, 2017; Cai y col., 2019).
Dentro de las cualidades que tienen los AO se considera el valor agronómico y, por lo tanto, el aporte nutrimental, microbiológico y atributos como el grado de madurez y/o toxicidad (Pérez y col., 2008). De manera que la caracterización de los $\mathrm{AO}$ es relevante porque aporta información sobre diferentes procesos edáficos físicos, químicos y biológicos, que se suscitan cuando estos se incorporan al suelo. Un AO rico en material recalcitrante, como lignina, puede ocasionar un mayor secuestro de carbono (C) respecto de abonos con compuestos más lábiles, como proteínas y materiales solubles en agua (Kumar y Goh, 1999); abonos con una relación carbono/nitrógeno $(\mathrm{C} / \mathrm{N})$ superior a 30 contribuyen a una inmovilización de $\mathrm{N}$ para el cultivo (Kumar y Goh, 1999; Marzi y col., 2019); una composta poco madura puede afectar la germinación de las semillas y el crecimiento y desarrollo de las plantas (Diacono y Montemurro, 2010; Tooba y col., 2014); asimismo, la aplicación de compostas que involucran estiércoles de animales como ovino, bovino, porcino, equino y gallinaza, que no alcanzaron las temperaturas adecuadas o cuyo proceso de elaboración fue corto, pueden provocar pérdida de nutrientes, como $\mathrm{N}$ en forma de $\mathrm{NH}_{3}$ o $\mathrm{NO}_{3}$, o por otra parte, la introducción de organismos patógenos como Escherichia coli o Salmonella spp. (Maffei y col., 2016). Además de las características de los AO, la cantidad y la frecuencia de aplicación, así como, el material de origen y su proceso de obtención (Félix-Herrán y col., 2008) tienen un papel importante. Pérez y col. (2008) examinaron diferentes $\mathrm{AO}$ y encontraron diversidad en $\mathrm{pH}$, $\mathrm{CE}, \mathrm{MO}$, carbono orgánico (CO) y $\mathrm{N}$ total, así como en la cantidad de hongos y levaduras, atribuibles a la cantidad y utilización diferencial del material de origen. Pérez y col. (2010) reportaron que las compostas de pollinaza tienen un alto aporte nutrimental, pero también pérdidas importantes de $\mathrm{N}$ por volatilización. Bashir y col. (2016), al analizar abonos como compostas de residuos sólidos municipales y estiércoles de aves, para conocer el impacto sobre los agregados del suelo y la estabilidad, detectaron una mejora en la formación de los macroagregados del suelo, después de 2 años de 
aplicación. Torres y col. (2016) caracterizaron AO y estimaron grandes riesgos de salinización cuando se emplean en zonas áridas, adjudicados a que el material para su elaboración tenía altas concentraciones de sodio.

De acuerdo con lo anterior, contribuir al conocimiento sobre las características de los AO permitirá proponer su uso adecuado y sugerir estrategias en sus procesos de obtención, para lograr la conservación y mantenimiento de suelos sanos como los destinados a cultivos florícolas.

Los AO del tipo composta designados a suelos florícolas son productos orgánicos obtenidos por un proceso biológico oxidativo, donde intervienen microrganismos para degradar los residuos orgánicos en un periodo promedio de 90 d; en vermicomposta, además de microorganismos intervienen en el proceso de degradación lombrices, y el tiempo de obtención es de $90 \mathrm{~d}$ - $210 \mathrm{~d}$; y en bocashi, se utilizan materiales diferentes que en la composta, como levadura, pulque o microorganismos del suelo para acelerar el proceso, que en promedio es de $40 \mathrm{~d}$, y determinar los posibles efectos de su uso.

El objetivo de la presente investigación fue examinar abonos orgánicos destinados a suelos florícolas y determinar los posibles efectos de su uso.

\section{MATERIALES Y MÉTODOS}

La recolección de las muestras se llevó a cabo en 10 sistemas productivos del municipio de Villa Guerrero, en el Estado de México, los cuales fueron elegidos por la facilidad de acceso y disposición a colaborar. Se ubicaron en las coordenadas geográficas comprendidas entre los paralelos 18 $56^{\prime} 17.1^{\prime \prime} \mathrm{N}$ y 1859'40.7” N y los meridianos 99³9'00.3” W y 099³9'46.1” W e intervalo altitudinal de $2044 \mathrm{msnm}$ a $2233 \mathrm{msnm}$.

La información recabada incluyó el tipo de AO catalogado por el productor, materia prima para su elaboración, tiempo de obtención, cultivo, destino y dosis de aplicación. En todos los ca- sos se trató de material listo para ser utilizado. La toma de muestras se realizó en cinco puntos de la pila del AO, para obtener finalmente una muestra compuesta de aproximadamente $1 \mathrm{~kg}$, de acuerdo a la Norma Ambiental para el Distrito Federal (NADF-020.AMBT-2011), la cual se identificó y se guardó en bolsas tipo "ziploc" para su análisis en el Laboratorio de Química del Tecnológico de Estudios Superiores de Villa Guerrero, en el Estado de México, México. Se secó en sombra, a temperatura ambiente promedio de $25^{\circ} \mathrm{C}$ durante $3 \mathrm{~d}$, para posteriormente tamizar ( $2 \mathrm{~mm}$ ) con base a la Norma Mexicana (NMX-FF-109-SCFI-2008). Las determinaciones para caracterizar los $\mathrm{AO}$ se realizaron por triplicado, el pH y la $\mathrm{CE}$ se midieron en una relación abono: agua (1:5 p/v) con potenciómetro (Orion Star modelo A211, Iowa, USA). Para la cuantificación de nitratos $\left(\mathrm{NO}_{3}\right)$ se utilizó una relación 1:5 (p/v) en una disolución de $\mathrm{CaCl}_{2}$ 0.01M. La mezcla se agitó por 30 min en una placa magnética (Thermo-Scientific CIMAREC, Iowa, USA), enseguida se filtró usando papel Whatman No. 1 y en la solución filtrada se determinó el contenido de nitratos con tiras AquaCheck $\left(\mathrm{HACH}^{\circledR}\right.$, USA).

Para el contenido de MO se especificó primero el porcentaje de cenizas a partir de la calcinación de la muestra en una mufla (FELISA, Jalisco, México) a $550{ }^{\circ} \mathrm{C}$ durante $2 \mathrm{~h}$. El porcentaje de $\mathrm{CO}$ se obtuvo al dividir el porcentaje de MO entre 1.724; ambos procedimientos se hicieron como lo indica la Norma Mexicana NMX-FF-109-SCFI-2008. La relación C/N se calculó al dividir el $\mathrm{CO}$ entre el contenido de $\mathrm{N}$ orgánico total.

Para la cuantificación de $\mathrm{P}$ y K, la muestra se sometió primero en disolución de Mehlich (agua, acetato de amonio, ácido nítrico, ácido etilendiaminotetraacético y fluoruro de amonio). La determinación de $\mathrm{P}$ consistió en tomar $1 \mathrm{~mL}$ del extracto y agregar $22 \mathrm{~mL}$ de agua, más $1 \mathrm{~mL}$ de molibdato de amonio, y luego se cuantificó mediante un espectrofotómetro (Genesys 10s Vis Termo Scientific, USA). En el caso del K, se usaron $0.10 \mathrm{~mL}$ de la disolución extractora y se agregaron $10 \mathrm{~mL}$ de agua destilada, para lue- 
go medir mediante absorción atómica (Analyst 206 Perkin Elmer, USA). El N total se precisó por el método Kjeldahl, la muestra se sometió a una digestión ácida, para conseguir $\mathrm{N}$ amoniacal, posteriormente este fue liberado mediante una destilación con $\mathrm{NaOH}$, y finalmente se cuantificó por un análisis volumétrico de la Asociación de Químicos Agrícolas Oficiales (AOAC, por sus siglas en inglés: Association of Official Agricultural Chemists) (AOAC, 1990).

Se realizaron pruebas de fitotoxicidad mediante la germinación de semillas de rábano ( $R a-$ pahnus sativus L.) y maíz (Zea mays L.) (Haq y col., 2014). Para ello se obtuvieron extractos con agua destilada al $5 \%$ de cada uno de los 10 AO recolectados, con base en el método propuesto por Zucconi y Bertoldi (1987). El extracto obtenido se filtró en papel Whatman No. 1 y se guardó en vasos de plástico ámbar de $200 \mathrm{~mL}$ a temperatura ambiente. En cajas Petri (90 mm de diámetro) se colocó papel filtro y encima 10 semillas de cada especie, enseguida se aplicaron $10 \mathrm{~mL}$ de cada extracto. Por tipo de $\mathrm{AO}$ se tuvieron 3 réplicas para cada especie vegetal, bajo un diseño completamente aleatorizado, con un tratamiento control, donde no se utilizó extracto de $\mathrm{AO}$, únicamente agua destilada. Las semillas se mantuvieron a temperatura ambiente promedio de $25{ }^{\circ} \mathrm{C}$. El índice germinativo (IG) se cuantificó después de $48 \mathrm{~h}$ al dividir el número de semillas germinadas en cada $\mathrm{AO}$, entre el número de semillas germinadas en el control (Huerta y col., 2010).

La actividad microbiana se estimó mediante la liberación de $\mathrm{C}$ en forma de $\mathrm{C}-\mathrm{CO}_{2}$, que se genera como resultado de la descomposición de la MO por parte de los mismos microorganismos, para ello se mezclaron $100 \mathrm{~g}$ de suelo con 2 g de 3 diferentes AO: bocashi (B1 y B2), composta (C1, C2, C3, C4, C5, C6 y C7) y lombricomposta (LC). Las mezclas se mantuvieron en frascos herméticos de vidrio con capacidad de $1 \mathrm{~L}$, a una humedad edáfica de $55 \%$ de espacio poroso lleno de agua y a una temperatura de $26^{\circ} \mathrm{C} \pm 1{ }^{\circ} \mathrm{C}$ en una estufa (ELVEC modelo 954-2, México D. F., México) bajo un diseño completamente al azar. El $\mathrm{C}-\mathrm{CO}_{2}$ retenido en una solución de $\mathrm{NaOH}$ se determinó por titulación mediante una disolución de $\mathrm{HCl} 0.05 \mathrm{M}$ (García y col., 2003). Las mediciones se realizaron diariamente durante $35 \mathrm{~d}$. El suelo que se utilizó se obtuvo de un sistema productivo de rosa (Rosa hybrida) de la misma zona, con una textura franco arenosa, con $66 \%, 28 \%$ y $6 \%$ de arena, limo y arcilla, respectivamente, un $\mathrm{pH}$ de 6.6, MO de $2.11 \%$, contenido de $\mathrm{P}$ de 145 ppm, K de 1200 ppm, Ca de 2500 ppm y $\mathrm{Mg}$ de 1600 ppm.

En la caracterización de los AO se obtuvo el promedio y el coeficiente de variación para cada una de las variables evaluadas. El IG y la respiración microbiana se evaluaron bajo un diseño completo al azar con tres repeticiones. Se especificó la homogeneidad de varianza entre tratamientos y la distribución normal de las observaciones de cada tratamiento (prueba Shapiro Wilk, $\mathrm{P}<0.05$ ), posteriormente se realizó un análisis de varianza y una comparación de medias de mínima diferencia significativa (MDS, $\mathrm{P}<0.05)$. Para cuantificar las tasas de respiración microbiana se utilizó una regresión lineal. Los datos se examinaron con el paquete estadístico InfoStat Versión 2016 (Di-Rienzo y col., 2016).

\section{RESULTADOS Y DISCUSIÓN}

El tipo de AO más abundante fue la composta (70 \%), aunque algunos floricultores llegan a elaborar bocashi (20\%) y, en menor cantidad, lombricomposta (10 \%) (Tabla 1). La preparación de los AO se realiza a partir de los residuos, según la especie florícola que se cultive en el sistema. Estos pueden incluir residuos de rosal, helechos y crisantemos, entre otros, mezclados con estiércol, principalmente de equino y bovino, de acuerdo a la información de los productores de donde se tomaron las muestras. La dosis de aplicación de los AO varió desde $10 \mathrm{~T} /$ ha a $100 \mathrm{~T} / \mathrm{ha}$, según el criterio del floricultor. Estas diferencias, sumadas a las características propias de cada AO y al tiempo de obtención, que puede ser hasta de 195 d de diferencia entre uno y otro, hicieron que su impacto en los cultivos fuese diferente (Tabla 1 ). 
Tabla 1. Abonos orgánicos y dosis de aplicación en suelos florícolas en Villa Guerrero, Estado de México.

Table 1. Organic fertilizers and application rates in floricultural soils in Villa Guerrero, Estado de Mexico.

\begin{tabular}{|c|c|c|c|c|}
\hline $\begin{array}{l}\text { Abono } \\
\text { orgánico }\end{array}$ & Componentes & $\begin{array}{l}\text { Tiempo } \\
\text { estimado de } \\
\text { obtención } \\
\text { (d) }\end{array}$ & $\begin{array}{l}\text { Dosis de } \\
\text { aplicación } \\
\text { (T/ha) }\end{array}$ & $\begin{array}{c}\text { Cultivos en donde se } \\
\text { aplica }\end{array}$ \\
\hline Bocashi (B1) & $\begin{array}{l}\text { Cascarilla de arroz, estiércol } \\
\text { equino, tierra, carbón, } \\
\text { melaza, suero de leche y } \\
\text { levadura. }\end{array}$ & 40 & 22 & $\begin{array}{c}\text { Helecho de } \\
\text { cuero (Rumohra } \\
\text { adiantiformis), } \\
\text { ruscus italiano } \\
\text { (Ruscus aculeatus) } \\
\text { hortensias (Hydrangea } \\
\text { macrophylla) }\end{array}$ \\
\hline Bocashi (B2) & $\begin{array}{l}\text { Cascarilla de arroz, estiércol } \\
\text { equino, carbón, melaza, } \\
\text { suero de leche y levadura. }\end{array}$ & 40 & 22 & $\begin{array}{c}\text { Helecho de } \\
\text { cuero (Rumohra } \\
\text { adiantiformis), } \\
\text { ruscus italiano } \\
\text { (Ruscus aculeatus) } \\
\text { hortensias (Hydrangea } \\
\text { macrophylla) }\end{array}$ \\
\hline Composta (C1) & $\begin{array}{l}\text { Desecho de helecho de cuero } \\
\text { (Rumohra adiantiformis), } \\
\text { estiércol equino, suero de } \\
\text { leche, levadura y melaza. }\end{array}$ & 180 & 22 & $\begin{array}{c}\text { Helecho de } \\
\text { cuero (Rumohra } \\
\text { adiantiformis), } \\
\text { ruscus italiano } \\
\text { (Ruscus aculeatus) } \\
\text { hortensias (Hydrangea } \\
\text { macrophylla) }\end{array}$ \\
\hline Composta (C2) & $\begin{array}{c}\text { Desecho de rosal (Rosa } \\
\text { hybrida), estiércol de bovino } \\
\text { y tierra. }\end{array}$ & 90 & 10 & Rosa hybrida \\
\hline Composta (C3) & $\begin{array}{l}\text { Rastrojo de rosal, estiércol de } \\
\text { bovino, melaza, urea y cal. }\end{array}$ & 15 & 20 & Rosa hybrida \\
\hline Composta (C4) & $\begin{array}{l}\text { Desecho de rosa (Rosa } \\
\text { hybrida), estiércol de bovino, } \\
\text { suero de leche y melaza. }\end{array}$ & 60 & 50 & Rosa hybrida \\
\hline Composta (C5) & $\begin{array}{l}\text { Desecho de rosa (Rosa hy- } \\
\text { brida), crisantemos, gerbera, } \\
\text { estiércol de bovino y tierra. }\end{array}$ & 90 & 10 & Rosa hybrida \\
\hline Composta (C6) & $\begin{array}{l}\text { Desechos de gerbera (Ger- } \\
\text { bera jamesonii), crisantemo } \\
\text { (Dendrathema morifoliumm), } \\
\text { hortensias (Hydrangea ma- } \\
\text { crophylla), helecho de cuero } \\
\text { (Rumohra adiantiformis) y } \\
\text { rosa (Rosa hybrida), estiércol } \\
\text { de bovino y melaza. }\end{array}$ & 210 & 100 & $\begin{array}{c}\text { Crisantemo } \\
\text { (Dendrathema } \\
\text { morifoliumm), rosa } \\
\text { (Rosa hybrida), gerbera } \\
\text { (Gerbera jamesonii), } \\
\text { hortensias (Hydrangea } \\
\text { macrophylla) y helecho } \\
\text { de cuero (Rumohra } \\
\text { adiantiformis) }\end{array}$ \\
\hline
\end{tabular}




\begin{tabular}{|l|c|c|c|c|}
\hline Composta (C7) & $\begin{array}{c}\text { Residuos de rosal (Rosa hy- } \\
\text { brida), gallinaza y melaza. }\end{array}$ & 60 & 30 & $\begin{array}{c}\text { Hortalizas, flores (Rosa } \\
\text { hybrida) }\end{array}$ \\
$\begin{array}{l}\text { Lombricomposta } \\
(\mathrm{LC})\end{array}$ & $\begin{array}{c}\text { Estiércol equino, composta } \\
\text { de desechos de mercado } \\
\text { (frutas y verduras) }\end{array}$ & 150 & 20 & $\begin{array}{c}\text { Helecho de } \\
\text { cuero (Rumohra } \\
\text { adiantiformis), } \\
\text { ruscus italiano } \\
\text { (Ruscus aculeatus), } \\
\text { hortensias (Hydrangea } \\
\text { macrophylla) }\end{array}$ \\
\hline
\end{tabular}

En el caso de la C3, aunque el proceso de compostaje es corto como un bocashi, e incompleto, los floricultores lo llegan a utilizar así. Se denominó como compostaje, porque no se agregaron materiales que se utilizan para el bocashi (cascarilla de arroz, levadura, pulque cenizas, entre otros). En el caso de la $\mathrm{C} 4 \mathrm{y} \mathrm{C}$, se consideraron compostas porque aunque intentan acelerar el proceso fermentativo con suero de leche y melaza o gallinaza y melaza, no incorporan productos que caracteriza a un bocashi (cascarilla de arroz, cenizas o carbón vegetal, entre otros).

Los $\mathrm{pH}$ de los $\mathrm{AO}$ presentaron un valor promedio de 8.23 y un coeficiente de variación de $87 \%$ (Tabla 2). Esta variación tan grande se atribuye a que se elaboran con diferentes materiales de origen y, sobre todo, porque los tiempos de obtención y almacenamiento los establece el floricultor. Los valores de $\mathrm{pH}$ registrados ( 7 a 8.5) en los AO indicaron que pasaron por un proceso de degradabilidad aerobia probablemente insuficiente, ya que idealmente, el pH esperado después de un proceso de compostaje debe ser de neutro a ligeramente básico, por lo que es posible que los procesos para la elaboración de AO para este estudio estén relativamente incompletos, probablemente porque la etapa de enfriamiento final, donde se reinicia la actividad microbiana mesofílica (Jiang y col., 2015; Montalvo y col., 2018), no se presenta o es muy corta. La utilización de los AO caracterizados no representa un problema a mediano plazo, ya que el $\mathrm{pH}$ que predomina en los suelos de la zona de estudio, del tipo andosol, es ácido, con valores de $\mathrm{pH}$ de alrededor de 6, como lo re- portan Guadarrama-Nonato y col. (2018); Yáñez-Ocampo y col. (2020). En el caso de LC, el valor de $\mathrm{pH}$ fue 7 , por lo que es ideal su utilización. En su mayoría, los AO presentaron valores dentro de lo permitido por la Norma $\mathrm{Me}$ xicana (NMX-FF-109-SCFI-2008) (5.5 a 8.5).

Los valores de CE se detectaron en un intervalo de $0.15 \mathrm{dS} / \mathrm{m}$ a $7 \mathrm{dS} / \mathrm{m}$, es decir, presentaron una variabilidad muy alta, lo que explica la elevada desviación estándar (DS) y coeficiente de variación (CV) (Tabla 2), atribuibles al material de origen y al proceso de obtención del AO. En el primer caso, la fuente de $\mathrm{N}$ que emplea cada floricultor para elaborarlo es un tipo de estiércol (ovino, equino o gallinaza) que a su vez está influenciado por la clase de alimentación, la edad del animal y la cantidad que se utiliza (Torres y col., 2016). En cuanto a la variabilidad vinculada a la obtención del AO, depende de la cantidad a usar y del tiempo de compostaje, ya que, por ejemplo, valores de CE altos dependen del tiempo de formación y precipitación de sales, como son iones amonio y carbonatos (Montalvo y col., 2018). De igual forma, el proceso de mineralización de la MO implica la liberación de sales como cationes $\mathrm{Na}^{+}, \mathrm{K}^{+}, \mathrm{Ca}^{2+}, \mathrm{y} \mathrm{Mg}^{2+}$ y aniones como $\mathrm{HCO}_{3}^{-}$, Cl- y $\mathrm{SO}_{2}-$, los cuales pueden conformar el $98 \%$ del total de las sales solubles que están presentes en un AO (Huerta y col., 2010). La CE tiene gran importancia cuando se aplican al terreno, ya que está relacionada con cantidad excesiva de sales en el suelo y, en consecuencia, puede limitar procesos fisiológicos, principalmente vinculados a la absorción de agua y nutrimentos, alteración en la tasa fotosintética, la transpiración y, por 
Tabla 2. Valores promedio de $\mathrm{pH}$, conductividad eléctrica (CE), nitratos $\left(\mathrm{NO}_{3}\right)$, materia orgánica (MO) y relación carbono/nitrógeno $(\mathrm{C} / \mathrm{N})$ en abonos orgánicos aplicados a suelos florícolas de Villa Guerrero, Estado de México.

Table 2. Averages values of $\mathrm{pH}$, electrical conductivity (EC), nitrates $\left(\mathrm{NO}_{3}\right)$, organic matter (OM) and carbon/nitrogen ratio $(\mathrm{C} / \mathrm{N})$ in organic fertilizers applied to floricultural soils of Villa Guerrero, Estado de Mexico.

\begin{tabular}{|l|c|c|c|c|c|}
\hline \multicolumn{1}{|c|}{ Abono orgánico } & $\mathbf{p H}$ & $\mathbf{C E}(\mathbf{d S} / \mathbf{m})$ & $\mathbf{N O}_{\mathbf{3}}(\mathbf{m g} / \mathbf{k g})$ & $\mathbf{M O}(\%)$ & $\mathbf{C} / \mathbf{N}$ \\
\hline B1 & $8.38 \pm 0.03$ & $0.20 \pm 0.01$ & $1.0 \pm 0$ & $28.90 \pm 0.67$ & $29.19 \pm 1.2$ \\
\hline B2 & $9.60 \pm 0.02$ & $0.48 \pm 0.01$ & $2.5 \pm 0$ & $70.34 \pm 0.53$ & $69.74 \pm 3.1$ \\
\hline C1 & $7.34 \pm 0.11$ & $0.49 \pm 0.01$ & $75 \pm 0$ & $80.08 \pm 2.14$ & $93.89 \pm 10.1$ \\
\hline C2 & $9.28 \pm 0.02$ & $1.25 \pm 0.20$ & $100 \pm 0$ & $62.24 \pm 1.73$ & $16.26 \pm 0.8$ \\
\hline C3 & $7.79 \pm 0.06$ & $6.70 \pm 0.38$ & $250 \pm 0$ & $75.20 \pm 1.31$ & $13.73 \pm 0.4$ \\
\hline C4 & $8.82 \pm 0.02$ & $3.80 \pm 1.10$ & $250 \pm 0$ & $58.33 \pm 0.75$ & $39.24 \pm 0.4$ \\
\hline C5 & $7.58 \pm 0.05$ & $0.15 \pm 0.02$ & $15 \pm 0$ & $28.80 \pm 5.85$ & $11.11 \pm 0.6$ \\
\hline C6 & $8.36 \pm 0.02$ & $4.18 \pm 0.20$ & $250 \pm 0$ & $31.79 \pm 0.95$ & $28.87 \pm 0.7$ \\
\hline C7 & $8.49 \pm 0.09$ & $2.48 \pm 0.40$ & $83 \pm 0$ & $48.37 \pm 11.6$ & $31.76 \pm 9.3$ \\
\hline LC & $7.17 \pm 0.03$ & $0.75 \pm 0.04$ & $100 \pm 0$ & $33.94 \pm 1.12$ & $19.71 \pm 0.3$ \\
\hline Promedio & 8.23 & 1.9 & 1.19 & 51.8 & 35 \\
\hline DS & 0.81 & 2.20 & 101.84 & 48.37 & 31.76 \\
\hline CV (\%) & 87 & $>100$ & 71 & 20 & 73 \\
\hline
\end{tabular}

$\mathrm{B}=$ bocashi, $\mathrm{C}=$ composta y LC = lombricomposta.

ello, afectar el desarrollo y crecimiento de la planta (Leogrande y Vitti, 2018). De igual forma, en el curso de germinación la $\mathrm{CE}$ impacta, porque valores altos pueden causar daños físicos, como quemaduras en plántulas, o impedir directamente el desarrollo de la radícula (Kebrom y col., 2019).

Aunque mayoritariamente los AO analizados no suponen un riesgo por efecto de una posible acumulación de sales, hubo $4 \mathrm{AO}$ que registraron valores de $\mathrm{CE}$ por arriba de $2.13 \mathrm{dS} / \mathrm{m}$ (Tabla 2), que según Torres y col. (2016) constituyen un riesgo potencial de salinización del suelo a largo plazo, sin embargo, de acuerdo a la Norma Mexicana (NMX-FF-109-SCFI-2008), la mayoría de los $\mathrm{AO}$ presentan una $\mathrm{CE}$ permitida (4 dS/m).

Como resultado de la heterogeneidad en la materia prima que utilizan los floricultores para la elaboración de los $\mathrm{AO}$, conjuntamente con el tiempo que dura el proceso, que incluye periodos de 15 d a 210 d (Tabla 1), los porcentajes de MO variaron de $28 \%$ a $80 \%$ ( Tabla 2 ), con un coeficiente de variación relati- vamente bajo (20 \%). En este sentido, el porcentaje de MO presente en cualquiera de los AO resulta benéfica, ya que en los suelos florícolas de la zona, su contenido se halla dentro de una clasificación agronómica mediana (Guadarrama-Nonato y col., 2018; Yáñez-Ocampo y col., 2020). Sin embargo, lo ideal es que en los AO la MO sea mayor al $40 \%$ pero menor al $60 \%$, de no ser así, significa que el proceso de compostaje puede estar incompleto, debido a que la MO no está siendo degradada, pero no necesariamente indica que el resto de sus atributos del AO no permitan su utilización para los cultivos. Cuando el tiempo de elaboración es de 90 d o superior, como se observa en cinco de los abonos caracterizados ( 1 1, C2, C5, C6, y LC), se considera que la MO que se obtiene es estable, porque se ha dado el tiempo suficiente para que se mineralice (Vaca y col., 2006); contrariamente, procesos de elaboración de poco tiempo, como sería el C3, hecho con rastrojo de rosal, generan MO lábil, con una degradabilidad incompta. La Norma Mexicana (NMX-FF-109-SCFI-2008) fija la cantidad ideal de aporte de $\mathrm{MO}$ de $20 \%$ a $50 \%$, intervalo que abarcan el $60 \%$ de los AO caracteriza- 
dos en este trabajo. El exceso de $\mathrm{MO}$ presente en algunos de los abonos orgánicos es indicativo de un proceso fermentativo incompleto, ya que normalmente gran parte del $\mathrm{CO}$ se pierde en forma de $\mathrm{CO}_{2}$. Por otra parte, los $\mathrm{AO}$ con exceso de $\mathrm{MO}$ como $\mathrm{B} 2$ y $\mathrm{C} 1$ se asocian a relaciones $\mathrm{C} / \mathrm{N}$ también muy altas, lo que implica que cuando se incorporan al suelo puede haber una inmovilización del $\mathrm{N}$ por parte de los microorganismos y hasta después de cierto tiempo alcanzar el equilibrio en el sistema edáfico.

La relación $\mathrm{C} / \mathrm{N}$ en promedio fue de 35 ( $\mathrm{Ta}-$ bla 2). Los $\mathrm{AO}$ que presentan más de 20 pueden tener un efecto negativo, debido a la inmovilización de $\mathrm{N}$ por los microorganismos como bacterias y hongos, además, es indicativo de que son ricos todavía en elemento vegetal difícil de degradar (Montalvo y col., 2018), como podría ser la parte leñosa de la rosa, que queda como componente principal en el producto final y que estaría conformado básicamente por material lignocelulósico. A su vez, la relación $\mathrm{C} / \mathrm{N}$ dependerá mucho también de la dinámica de $\mathrm{N}$, como la pérdida de $\mathrm{N}-\mathrm{NH}_{3}$, la formación de $\mathrm{N}-\mathrm{NO}_{-}$y el comportamiento de $\mathrm{N}-\mathrm{HH}_{4}$ a lo largo del proceso de compostaje (Montalvo y col., 2018). Con base en la Norma Mexicana (NMX-FF-109-SCFI-2008), únicamente 4 de los $\mathrm{AO}$ tienen un valor de $\mathrm{C} / \mathrm{N}$ adecuado $(<20)$, que corresponde a las compostas C2 y C5 y la LC. Los bocashi y las compostas obtenidas en tiempos cortos (C3) e intermedios ( $\mathrm{C} 4 \mathrm{y} \mathrm{C}$ 7) presentaron valores altos de $\mathrm{C} / \mathrm{N}$, indicativo de que los procesos fermentativos pueden estar incompletos. Las compostas tuvieron un alto contenido de nitratos $\left(\mathrm{NO}_{3}\right)$ con una diferencia de más de 100 unidades respecto de los bocashi, esto se atribuye a las diferencias del proceso. Como lo menciona Cáceres y col. (2017) en las compostas aún en estado de maduración, los microorganismos mesófilos siguen trabajando, ocasionando la formación de $\mathrm{NO}_{3}$, además, factores como la humedad y oxigenación también pueden intervenir, una humedad adecuada favorece la entrada de oxígeno y la formación de $\mathrm{NO}_{3}$, situación que el floricultor controla conforme a su experiencia.

En cuanto al aporte de N, K y P (Tabla 3), el contenido de $\mathrm{N}$ promedio fue similar al de otros $\mathrm{AO}$, pero el $\mathrm{P}$ y el $\mathrm{K}$ es menor al reportado por Álvarez-Sánchez y col. (2006); Angelova y col. (2013); Ávalos y col. (2018). Conforme a la Norma Mexicana (NMX-FF-109-SCFI-2008), el N debe estar entre $1 \%$ y $4 \%$, y el promedio obte-

- Tabla 3. Valores promedio de nitrógeno $(\mathrm{N})$, fósforo $(\mathrm{P})$ y potasio $(\mathrm{K})$ en abonos orgánicos aplicados a suelos florícolas de Villa Guerrero, Estado de México.

Table 3. Average values of nitrogen (N), phosphorus (P) and potassium (K) in organic fertilizers applied to floricultural soils of Villa Guerrero, Estado de Mexico.

\begin{tabular}{|c|c|c|c|}
\hline \multirow{2}{*}{ Abono orgánico } & \multirow{2}{*}{$\underset{(\%)}{\mathbf{N}}$} & $\mathbf{P}$ & $\mathbf{K}$ \\
\hline & & \multicolumn{2}{|c|}{ (mg/kg) } \\
\hline B1 & $0.57 \pm 0.02$ & $88.86 \pm 0.49$ & $1000 \pm 0.36$ \\
\hline B2 & $0.59 \pm 0.02$ & $135.77 \pm 0.40$ & $1000 \pm 0.45$ \\
\hline $\mathrm{C} 1$ & $0.50 \pm 0.06$ & $98.27 \pm 0.00$ & $600 \pm 0.32$ \\
\hline $\mathrm{C} 2$ & $2.22 \pm 0.05$ & $177.42 \pm 0.33$ & $3200 \pm 0.76$ \\
\hline C3 & $3.18 \pm 0.05$ & $343.98 \pm 0.53$ & $4400 \pm 0.32$ \\
\hline C4 & $0.86 \pm 0.05$ & $194.12 \pm 0.62$ & $3200 \pm 0.52$ \\
\hline C5 & $1.51 \pm 0.05$ & $182.29 \pm 2.63$ & $200 \pm 0.18$ \\
\hline C6 & $0.64 \pm 0.00$ & $186.96 \pm 0.73$ & $3000 \pm 0.22$ \\
\hline $\mathrm{C} 7$ & $0.89 \pm 0.07$ & $334.41 \pm 4.96$ & $800 \pm 0.53$ \\
\hline $\mathrm{LC}$ & $1.00 \pm 0.05$ & $192.00 \pm 1.17$ & $400 \pm 0.31$ \\
\hline Promedio & 1.19 & 193.41 & 1789 \\
\hline DS & 0.87 & 85.81 & 258.19 \\
\hline CV (\%) & 71 & 44 & 84 \\
\hline
\end{tabular}

$\mathrm{N}=3 \pm \mathrm{DS}$ (desviación estándar), $\mathrm{B}=$ bocashi, $\mathrm{C}=$ composta y LC = lombricomposta. 
nido en este estudio se ubica en ese intervalo, de manera que la aplicación de este tipo de AO es benéfica para cualquier cultivo florícola.

Respecto a las pruebas de fitotoxicidad en la germinación de las semillas de rábano, el IG indicó que hubo diferencias estadísticas significativas $(\mathrm{P}<0.05)$; únicamente el $\mathrm{B} 2$ y la C3 tuvieron mayor germinación de semillas que el control (Figura 1a). Estos resultados muestran que el $80 \%$ de los lixiviados no tienen un efecto provechoso en la germinación de semillas de rábano. El IG en semillas de maíz también difirió significativamente $(\mathrm{P}<0.05)$, pero, en contraste, en las semillas de rábano el IG fue en el $70 \%$ de los $\mathrm{AO}>1$ (Figura 1b), lo que denota que la aplicación de lixiviados favoreció la germinación. Los resultados del IG en el caso de maíz coin- ciden con lo encontrado por Arancon y col. (2012), quienes reportan una germinación mayor cuando se utilizan extractos de composta respecto del testigo (agua destilada), atribuido a que en el extracto o lixiviado encuentran ácidos húmicos, fitohormonas o metabolitos secundarios que se considera que promueven la germinación. En este estudio se observó que las semillas de maíz fueron estimuladas en su germinación (Figura 1b), lo cual también se logró en semillas de Vignia ungiculata (Arunbabu y col., 2017) y de trigo (Ameen, 2020).

La actividad microbiana, evaluada a partir de la liberación de $\mathrm{C}-\mathrm{CO}_{2}$, indicó una mayor cantidad acumulada al mezclar suelo con C3, y fue diferente significativamente respecto de B1, LC y del suelo control $(\mathrm{P}<0.05)$ (Tabla 4). Este tipo de composta tiene una relación $\mathrm{C} / \mathrm{N}$ baja

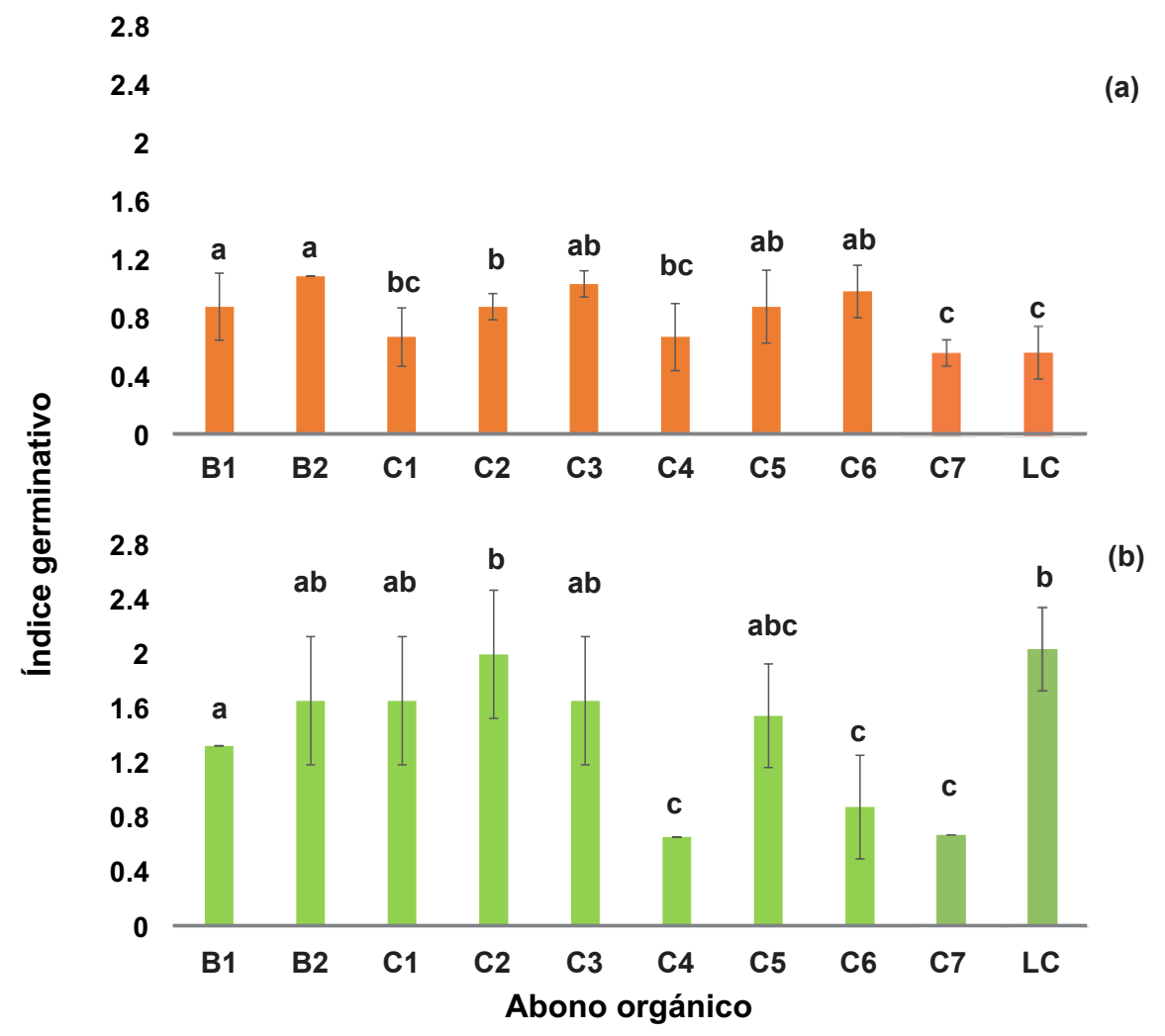

Figura 1. Comparación de medias (Mínima diferencia significativa, MDS, $\alpha=0.05$ ) del índice germinativo en rábano (Rapahanus sativus) (a) y maíz (Zea mays) (b), a $5 \%$ de concentración de lixiviados obtenidos de abonos orgánicos. $\mathrm{B}=$ bocashi, $\mathrm{C}=$ composta y $\mathrm{LC}=$ lombricomposta.

Figure 1. Comparison of means (Least significant difference, LSD, $\alpha=0.05$ ) of the germination index in radish (Rapahanus sativus) (a) and corn (Zea mays) (b), at $5 \%$ concentrations of leachates obtained from organic fertilizers. $\mathrm{B}=$ bocashi, $\mathrm{C}=$ compost and $\mathrm{LC}=$ vermicompost. 
Tabla 4. $\mathrm{C}-\mathrm{CO}_{2}$ liberado acumulado durante $35 \mathrm{~d}$ en incubaciones aerobias de mezcla de suelo con tres diferentes abonos orgánicos utilizados en suelos florícolas de Villa Guerrero, Estado de México. Table 4. Cumulative $\mathrm{C}-\mathrm{CO}_{2}$ during $35 \mathrm{~d}$ in aerobic incubations of soil mixture with three different organic fertilizers used in floricultural soils of Villa Guerrero, Estado de Mexico.

\begin{tabular}{|c|c|c|c|c|}
\hline Abono orgánico & $\begin{array}{l}\text { C-CO acumulado } \\
\text { (mg/kg suelo) }\end{array}$ & $\begin{array}{c}\mathrm{C}-\mathrm{CO}_{2} \text { acumulado } \\
\text { respecto del suelo } \\
\text { (\%) }\end{array}$ & $\begin{array}{c}\text { Ajuste a modelo } \\
\text { lineal } \\
\left.\text { (mg/kg de C-CO } \mathrm{CO}_{2} / \mathrm{d}\right)\end{array}$ & $\mathbf{R}^{2}$ \\
\hline C3 & $503.45 \pm 16.43^{\mathrm{a}}$ & 22.03 & $13.10 x+86.9$ & 0.9650 \\
\hline B1 & $417.09 \pm 36.32^{b}$ & 1.10 & $10.30 x+73.0$ & 0.9826 \\
\hline $\mathrm{LC}$ & $460.73 \pm 39.93^{\mathrm{ab}}$ & 11.67 & $11.62 x+88.4$ & 0.9585 \\
\hline Suelo & $412.55 \pm 42.28^{b}$ & & $10.35 x+75.08$ & 0.9621 \\
\hline MDS & 66.35 & & & \\
\hline
\end{tabular}

$\mathrm{N}=3 \pm \mathrm{DS}$ (desviación estándar), letras diferentes indican diferencias significativas $(\mathrm{P}<0.05)$, MDS = mínima diferencia significativa, $\mathrm{B}=$ bocashi, $\mathrm{C}=$ composta y $\mathrm{LC}=$ lombricomposta.

(13.73), lo que favorece que la MO sea accesible para los microorganismos. Por otra parte, $\mathrm{B} 1$ fue el $\mathrm{AO}$ que liberó más lentamente el C orgánico (10.30 mg/kg en 1 d). Es posible que la relación $\mathrm{C} / \mathrm{N}$ alta (29.19) haya contribuido a una baja actividad microbiana. En este sentido, los resultados de este estudio contrastan con el de Agüero y Terry (2014), quienes indicaron que bocashi tiene una aportación importante de carga microbiana cuando se incorpora al suelo, por lo que se esperaría una mayor liberación de $\mathrm{C}-\mathrm{CO}_{2}$. El $\mathrm{C}-\mathrm{CO}_{2}$ liberado al aplicar $\mathrm{C} 3$ es indicativo de que hubo actividad microbiana. Esta composta elevó en un $22 \%$ la liberación de $\mathrm{C}-\mathrm{CO}_{2}$ respecto al suelo control, lo que resultaría benéfico, porque implica la liberación de nutrimentos que, en condiciones de campo, el cultivo puede aprovechar. El análisis de regresión lineal indica que las tasas de liberación de $\mathrm{C}-\mathrm{CO}_{2}$ fueron más altas cuando se aplicó C3 y LC (11.6 mg/kg a $13.10 \mathrm{mg} / \mathrm{kg}$ de $\mathrm{C}-\mathrm{CO}_{2} / \mathrm{d}$ ), mientras que la tasa de mineralización al aplicar B1 es menor y similar al suelo al que no se aplicó AO (Tabla 4). Cabe mencionar que la biomasa microbiana actúa sobre la MO del suelo (Sarmiento y col., 2019) y sobre la que se incorpora en el propio abono, en donde normalmente predominan actinomicetos que degradan MO lábil (Yáñez-Ocampo y col., 2020). El hecho de que la C3 mostró una mayor respiración microbiana es indicativo de que hay una mayor cantidad de sustrato para ser utilizado por los microorganismos, asociado a la presencia de un material lábil (Carabassa y col., 2020), e indirectamente también indica poca cantidad de sustrato que los microorganismos pueden utilizar (Fernández y col., 2010); ambas características son importantes de considerar al momento de aplicarlos en los cultivos. De manera que la incorporación de MO al suelo promoverá la actividad microbiana, pero depende mucho de su calidad. Cada AO generará una respuesta diferente, atribuida al grado de madurez, relación $\mathrm{C} / \mathrm{N}, \mathrm{MO}$ lábil, aporte de nutrientes, entre otras características.

\section{CONCLUSIONES}

Los resultados indicaron que los abonos orgánicos preparados por los floricultores y estudiados en la presente investigación, son preferentemente de tipo composta. Sus características físicas, químicas y biológicas muestran una gran variabilidad, atribuida principalmente a la heterogeneidad del material de origen y al tiempo de elaboración. Es importante señalar que en algunos casos hay necesidad de completar el proceso fermentativo para asegurar relaciones carbono/nitrógeno adecuados. La conductividad eléctrica, potencial de hidrógeno y contenido de nitrógeno fueron las características que mayor diversidad mostraron. Los abonos orgánicos evaluados no presentan un riesgo 
al aplicarlos a los suelos florícolas, por el contrario, aportan mínimamente $10 \%$ de materia orgánica, lo que significa una ventaja para im- pulsar la actividad microbiana y mantener las propiedades del suelo en este tipo de sistemas productivos.

\section{REFERENCIAS}

Agüero, D. y Terry. E. (2014). Revisión bibliográfica. Generalidades de los abonos orgánicos: importancia del bocashi como alternativa nutricional para suelos y plantas. Cultivos Tropicales. 35(4): 52-59.

Álvarez-Sánchez, E., Vázquez-Alarcón, A., Castellanos, J. Z. y Cueto-Wong, J. (2006). Efectividad biológica de abonos orgánicos en el crecimiento de trigo. Terra Latinoamericana. 24(2): 261-268.

Ameen, A. (2020). Comparison of crop production efficiency of compost leachate with chemical fertilizer and evaluating its effect on germination and growth of wheat crop. African Journal of Biotechnology. 19(5): 282-286.

Angelova, V. R., Akova, V. I., Artinova, N. S., and Ivanov, K. I. (2013). The effect of organic amendments on soil chemical characteristics. Bulgarian Journal of Agricultural Science. 19(5): 958-971.

AOAC, Association of Official Agricultural Chemists (1990). Official Methods of Analysis. Association of Official Analytical Chemists 923.03. Washington: AOAC.115 Pp.

Arancon, N. Q., Pant, A., Radovich, T., Hue, N. V., Potter, J. K., and Converse, C. E. (2012). Seed germination and seedling growth of tomato and lettuce as affected by vermicompost water extracts (Teas). HortScience. 47:1722-1728.

Arunbabu, V., Indu, S. K., and Ramasamy, E. V. (2017). Arun Leachate pollution index as an effective tool in determining the phytotoxicity of municipal solid waste leachate. Waste Management. 68:329-336.

Ávalos, M., Figueroa, U., García, J. L., Vázquez, C., Gallegos, M. A. y Orona, I. (2018). Bioinoculantes y abonos orgánicos en la producción de maíz forrajero. Nova Scientia. 20(10): 170-189.

Bashir, K., Alil, S., Sohail, S., and Ahmad, I. (2016). Effect of organic amendments on distribution, stability and carbon concentration of soil aggregates. Pakistan Journal of Agricultural Science. 53(4): 955-961.

Bonanomi, G., De-Filippis, F., Zotti M., Idbellaa, M., Cesaranoa, G., Al-Rowailyc, S., and ElGawadc, A. (2020). Repeated applications of organic amendments promote beneficial microbiota, improve soil fertility and increase crop yield. Applied Soil Ecology. 156: 103714.

Cabrera, R. I., Solís-Pérez, A. R. y Gómez, G. (2017). Aplicaciones de nutrición vegetal en cultivos de flor de corte. En R. V. Flórez (Ed.), Consideraciones sobre producción, manejo y poscosecha de flores de corte con énfasis en rosa y clavel (pp. 33-47). Bogotá: Editorial Universidad Nacional de Colombia.

Cáceres, R., Malynska, K., and Marfá, O. (2017). Nitrification within composting: A review. Waste Management. 72: 119-137.

Cai, A., Xua, M., Wang, B., Zhang, W., Liang, G., Hou, E., and Luo, Y. (2019). Manure acts as a better fertilizer for increasing crop yields than synthetic fertilizer does by improving soil fertility. Soil \& Tillage Research. 189:168-175.

Carabassa, V., Domene, X., and Alcañiz, J. P. (2020). Soil restoration using compost-like-outputs and digestates from non-source-separated urban waste as organic amendments: Limitations and opportunities. Journal of Environmental Management. 255: 109909.

Diacono, M. and Montemurro, F. (2010). Long-term effects of organic amendments on soil fertility. A review. Agronomy for Sustainable Development. 30: 401-422.

Dikr, W. and Belete, K. (2017). Review on the effect of organic fertilizers, biofertilizers and inorganic fertilizers (NPK) on growth and flower yield of marigold (Targetes erecta L.). Academic Research Journal of Agricultural Science and Research. 5(3): 192-204.

Di-Rienzo, J. A., Casanoves, F., Balzarini, M. G., González, L., Tablada, M., and Robledo, C. W. (2016). InfoStat (versión 2016). [Software de cómputo]. Córdoba, Argentina: Grupo InfoStat, FCA, Universidad Nacional de Córdoba.

Félix-Herrán, J. A., Sañudo-Torres, R., Rojo-Martínez, G., Martínez-Ruiz, R. y Olalde-Portugal, V. (2008). Importancia de los abonos orgánicos. Ra Ximhai. 4(1): 57-67.

Fernández, R., Quiroga, A., Zorati, C., and Noellemeyer, E. (2010). Carbon contents and respiration rates of aggregate size fractions under no-till and conventional tillage. Soil \& Tillage Research.109(2): 103-109.

García, C., Gil, F., Hernández, T. y Trasar, C. (2003). Técnicas de análisis de parámetros bioquímicos en suelos: medida de las actividades enzimáticas y biomasa microbiana. España: Mundi-Prensa. 371 Pp.

Guadarrama-Nonato, A., Mejía-Carranza, J. y RamírezGerardo, M. G. (2018). Mineralización de la materia orgánica en suelos con manejo diferencial en cultivo de rosa. Acta Universitaria. 28(32): 33-41.

Haq, T., Ali, T., and Begun, R. (2014). Seed germination bioassay using maize seeds for phytoxicity evaluation of di- 
fferent composted materials. Pakistan Journal of Botany. 46(2): 539-542.

Huerta, O., López, M., and Soliva, M. (2010). Procés de compostatge caracterizació de mostres. Barcelona: Ed. Diputació de Barcelona. $432 \mathrm{Pp}$.

Jiang, J., Liu, X., Huang, Y., and Huang, H. (2015). Inoculation with nitrogen turnover bacterial agent appropriately increasing nitrogen and promoting maturity in pig manure composting. Waste Management. 39: 78-85.

Kebrom, T., Woldesenbet, S., Bayabil, H., Garcia, M., Gao, M., Ampim, P., ..., and Fares, A. (2019). Evaluation of phytotoxicity of three organic amendments to collard greens using the seed germination bioassay. Environmental Science and Pollution Research. 26(6): 5454-5462.

Kumar, K. and Goh, K. M. (1999). Crop residues and management practices: Effects on soil quality, soil nitrogen dynamics, crop yield, and nitrogen recovery. Advances in Agronomy. 68: 197-318.

Leogrande, R. and Vitti, C. (2018). Use of organic amendments to reclaim saline and sodic soils: a review. Arid land and Research and Management. 33(1):1-21.

Maffei, F., Batalhaa, E., Landgrafa, M., Schaffnerb, D. W., and Franco, B. (2016). Microbiology of organic and conventionally grown fresh produce. Brazilian Journal of Microbiology. 47(1): 99-105.

Marzi, M., Ahbazi, K., Kharazi, N., and Rezaei, M. (2019). The influence of organic amendment source on carbon and nitrogen mineralization in different soils. Journal of Soil Science and Plant Nutrition. 20:177-19.

Montalvo, P. A. J., Dongo, L. F. O., Maraví, J. L. C., Monzón, L. A. T., Coral, M. F. C. y Figueroa, L. V. (2018). Transformación del nitrógeno durante el compostaje de bosta de caballo. Revista Producción + Limpia. 13(22): 77-88.

NADF-020.AMBT-2011 (2011). Norma ambiental para el Distrito Federal. Secretaría de Medio Ambiente del Distrito Federal. [En línea]. Disponible en: http://data.sedema.cdmx. gob.mx/padla/images/stories/normatividaddf/nadf_020_ ambt_2011.pdf. Fecha de consulta: 20 de mayo de 2020.

NMX-FF-109-SCFI-2008 (2008). Norma mexicana humus de lombriz (lombricomposta) especificaciones y métodos de prueba. [En línea]. Disponible en: https://www.dof.gob.mx/ nota_detalle.php?codigo $=5044562 \&$ fecha $=10 / 06 / 2008$. Fecha de consulta: 6 de marzo de 2020.

Pérez, A., Céspedes, C. y Núñez, P. (2008). Caracterización física-química y biológica de enmiendas orgánicas aplicadas en la producción de cultivos en República Dominicana. R. C. Suelo. Nutrición Vegetal. 8(4): 10-29.

Pérez, C. R., Pérez C. A. y Vertel, M. M. (2010). Caracterización nutricional, fisicoquímica y microbiológica de tres abonos orgánicos para uso en agroecosistemas de pasturas en la subregión Sabanas del departamento de Sucre, Colombia. Revista Tumbaga.1(5):27-37.

Sakin, E., Celik, A., Dogan, Z., Yalcin, H., and Seyreck, A. (2018). Comparing carbon pools and some soil quality parameters of soils in organic and conventional agriculture land. Fresenius Environmental Bulletin. 27(11): 7536-7544.

Sarmiento, G. J., Amézquita, M. A. y Mena, L. M. (2019). Uso de bocashi y microorganismos eficaces como alternativa ecológica en el cultivo de fresa en zonas áridas. Scientia Agropecuaria.10(1): 55-61.

Scotti, R., Bonanomi, G., Scelza, R., Zoina, A., and Rao, M. (2015). Organic amendments as sustainable tool to recovery fertility in intensive agricultural systems. Journal of Soil Science and Plant Nutrition. 15(2): 333-352.

Tooba, H., Tasneem, A., and Razia, B. (2014). Seed germination bioassay using maize seeds for Phytoxicity evaluation of different composted materials. Pakistan Journal of Botany. 46: 539-542.

Torres, D., Mendoza, B., Marco, L. M. y Gómez, C. (2016). Riesgos de salinización y solidificación por el uso de abonos orgánicos en la depresión de Quíbor-Venezuela. Multiciencias. 16(2): 133-142.

Vaca, R., Lugo, J. y Esteller, A. (2006). Caracterización de la materia orgánica soluble y de los ácidos húmicos en suelo acondicionado con lodo residual fresco o compostado. Revista Internacional de Contaminación Ambiental. 22(1): 27-30.

Vilkienè, M., Ambrazaitienè, D., Karčauskienè, D., and Dabkevičius, Z. (2016). Assessment of soil organic matter mineralization under various management practices. Acta Agriculturae Scandinavica, Section B - Soil \& Plant Science. 66(8): 641-646.

Yáñez-Ocampo, G., Sánchez-González, M. E., De-la-Portilla-López, N., Marmolejo-Santillán, Y., Del-Águila-Juárez, P., Lugo-de-la-Fuente, J. y Vaca-Paulín, R. (2020). Densidad poblacional de actinomicetos en suelos florícolas, enmendados con vermicomposta. Terra Latinoamericana. 38: 745-753.

Zucconi, F. and Bertoldi, M. (1987). Compost specification for the production and characterization of compost from municipal solid waste. In M. de-Bertoldi, M. P. Ferranti, and F. Zucconi (Eds.), Compost: production, quality and use (pp. 30-50). Barking: Elsevier Applied Science Publishers. 